\title{
RESPONSE OF SCANNING FABRY-PÉROT INTERFEROMETER TO SPEED-DEPENDENT ASYMMETRIC VOIGT PROFILE
}

\author{
A. Bielski, S. Brym*, R. Ciunylo and J. Jurkowski \\ Institute of Plysics, Nicholas Copernicus University \\ Grudziądzka 5/7, 87-100 Toruí, Poland
}

(Received May 21, 1996; revised version July 17, 1996)

\begin{abstract}
A detailed analysis of the deviations from the Voigt profile caused by the correlation effects and collision-time asymmetry observed by means of a Fabry-Pérot interferometer is presented. An expression for the response of scanning Fabry-Pérot interferometer to the speed-dependent asymmetric Voigt profile is derived.
\end{abstract}

PACS numbers: $32.70 . \mathrm{Jz}, 33.70 . \mathrm{Jg}, 34.20 . \mathrm{Cf}$

\section{Introduction}

The Fabry-Pérot interferometer (FPI) is one of a few spectroscopic instruments for which analytic approximation to its instrumental function is known with a high accuracy. If the real shape of a spectral line is known, the shape observed by means of the FPI can be expressed as a convolution of the real shape and the instrumental function. The expression for such a convolution is called the response of the FPI. The responses of the scaming FPI to the Voigt profile (VP) [1], asymmetric Voigt profile (AVP) [2] and speed-dependent Voigt profile (SDVP) [3] have been reported in previous papers. In this paper we present a formula for the response of FPI to the speed-dependent asymmetric Voigt profile. We also analyse the dependence of the collision-time asymmetry on the emitter velocity and discuss the influence of the correlation effects and collision-time asymmetry on the deviations of the real profile from the Voigt profile observed using the Fabry-Pérot interferometer.

In the near-wing region the line shape of the pressure broadened spectral line can be approximately described by a superposition of the Lorentzian and dispersion profiles $[4,5]$ :

$$
I_{\mathrm{L}}(\omega)=\frac{1}{\pi} \frac{\gamma_{\mathrm{L}} / 2+\beta\left(\omega-\omega_{0}-\Delta\right)}{\left(\omega-\omega_{0}-\Delta\right)^{2}+\left(\gamma_{\mathrm{L}} / 2\right)^{2}}
$$

*Permanent address: Department of Pliysics, Pedagogical University, Żołnierska 14, 10-561 Olsztyn, Poland. 
where $\gamma_{L}$ is the Lorentzian width (FWIIM), $\Delta$ is the shift of the maximum with respect to the unperturbed frequency $\omega_{0}$ of the line and $\beta$ is the collision-time asymmetry. The collision-time asymmetry was the subject of both theoretical and experimental investigations [4-12].

If the Lorentzian width $\gamma_{\mathrm{L}}$, shift $\Delta$, and the asymmetry parameter $\beta$ are independent of the emitter velocity, then the resulting line shape, generated by the pressure and Doppler broadening, is given by an asymmetric Voigt profile $I_{\mathrm{AVP}}(\omega)$ which is a convolution of the asymmetric profile $I_{\mathrm{L}}(\omega), \mathrm{Eq} .(1)$, and the Gaussian distributions. This assumption is not correct in general case because all parameters of the pressure broadened line are dependent on the emitter velocity. Following Berman [13], in such a case the resulting profile should be written in the form

$$
I_{\mathrm{SDAVP}}(\omega)=\int \mathrm{d}^{3} v_{\mathrm{E}} f_{m_{\mathrm{E}}}\left(v_{\mathrm{E}}\right) I_{\mathrm{L}}\left(\omega-v_{\mathrm{E}} k\right) .
$$

IIere $k$ is the propagation vector of emitted radiation such that $k=\omega_{0} / c$ and $f_{m_{\mathrm{E}}}\left(v_{\mathrm{E}}\right)$ is the Maxwellian distribution of the emitter velocity $v_{\mathrm{E}}$ when gas temperature is $T$ and $m_{\mathrm{E}}$ is the mass of the radiating atoms. Following IIarris et al. [10] this profile will be referred to as the speed-dependent asymmetric Voigt profile (SDAVP). Such line profiles were experimentally and theoretically investigated by Lewis and his coworkers [10-12].

\section{Near-wing line shape parameter's}

In the classical limit of the unified Franck-Condon (UFC) theory $[4,5]$ the Lorentzian width $\gamma_{\mathrm{L}}\left(v_{\mathrm{E}}\right)$ and shift $\Delta\left(v_{\mathrm{E}}\right)$ for a given value $v_{\mathrm{E}}$ of the emitter velocity averaged over perturber velocities [14], can be expressed as

$$
\begin{aligned}
& \gamma_{\mathrm{L}}\left(v_{\mathrm{E}}\right)=8 \pi N \int \mathrm{d}^{3} v_{\mathrm{EP}} f_{m_{\mathrm{P}}}\left(v_{\mathrm{E}}+v_{\mathrm{EP}}\right) v_{\mathrm{EP}} \int_{0}^{+\infty} \rho \sin ^{2}\left[\eta\left(\rho, v_{\mathrm{EP}} ; \infty\right)\right] \mathrm{d} \rho \\
& \Delta\left(v_{\mathrm{E}}\right)=2 \pi N \int \mathrm{d}^{3} v_{\mathrm{EP}} f_{m_{\mathrm{P}}}\left(v_{\mathrm{E}}+v_{\mathrm{EP}}\right) v_{\mathrm{EP}} \int_{0}^{+\infty} \rho \sin \left[2 \eta\left(\rho, v_{\mathrm{EP}} ; \infty\right)\right] \mathrm{d} \rho
\end{aligned}
$$

where $v_{\mathrm{EP}}$ denotes the relative emitter-perturber velocity, which is connected with perturber velocity by equality $v_{\mathrm{P}}=v_{\mathrm{E}}+v_{\mathrm{EP}}$. Using a formula for the asymmetry parameter $\beta$ derived by Szudy and Baylis $[4,5]$ and performing an averaging over perturber velocities in analogy to a treatment proposed by Ward et al. [14] we obtain

$$
\begin{gathered}
\beta\left(v_{\mathrm{E}}\right)=8 \pi N \int \mathrm{d}^{3} v_{\mathrm{EP}} f_{m_{\mathrm{P}}}\left(v_{\mathrm{E}}+v_{\mathrm{EP}}\right) v_{\mathrm{EP}} \\
\times \int_{0}^{+\infty} \rho a\left(\rho, v_{\mathrm{EP}}\right) \sin \left[\eta\left(\rho, v_{\mathrm{EP}} ; \infty\right)\right] \mathrm{d} \rho
\end{gathered}
$$

where

$$
a\left(\rho, v_{\mathrm{EP}}\right)=\frac{1}{\hbar} \int_{0}^{\infty} t \Delta V[r(t)] \sin \left[\eta\left(\rho, v_{\mathrm{EP}} ; t\right)\right] \mathrm{d} t .
$$

In Eqs. (3)-(6) $\rho$ is the impact parameter, $N$ denotes the density number of the perturbing gas and $\eta\left(\rho, v_{\mathrm{EP}} ; t\right)$ is the phase shift expressed as a function of $\rho, v_{\mathrm{EP}}$ and time $t$. 
Assuming that the perturbers move along classical straight-line trajectories, $\eta\left(\rho, v_{\mathrm{EP}} ; t\right)$ can be written as

$$
\eta\left(\rho, v_{\mathrm{EP}} ; t\right)=\frac{1}{\hbar} \int_{0}^{t} \Delta V[r(\tau)] \mathrm{d} \tau
$$

where $\Delta V(r)$ is the difference of adiabatic potentials describing the interaction between the perturber and the emitting atom in its upper and lower state at the distance $r(\tau)=\sqrt{\rho^{2}+v_{\mathrm{EP}}^{2} \tau^{2}}$. The line profile parameters $\gamma_{\mathrm{L}}\left(v_{\mathrm{E}}\right), \Delta\left(v_{\mathrm{E}}\right)$ and $\beta\left(v_{\mathrm{E}}\right)$ averaged over all emitter velocities can be written as $\gamma_{\mathrm{L}}=\int \mathrm{d}^{3} v_{\mathrm{E}} f_{m_{\mathrm{E}}}\left(v_{\mathrm{E}}\right) \gamma_{\mathrm{L}}\left(v_{\mathrm{E}}\right)$, $\Delta=\int \mathrm{d}^{3} v_{\mathrm{E}} f_{m_{\mathrm{E}}}\left(v_{\mathrm{E}}\right) \Delta\left(v_{\mathrm{E}}\right), \beta=\int \mathrm{d}^{3} v_{\mathrm{E}} f_{m_{\mathrm{E}}}\left(v_{\mathrm{E}}\right) \beta\left(v_{\mathrm{E}}\right)$.

Following Ward et al. [14] we introduce now the dimensionless width

$$
B_{W}(x ; \alpha)=\frac{\gamma_{L}\left(x v_{m_{E}}\right)}{\gamma_{L}}
$$

shift

$$
B_{\mathrm{S}}(x ; \alpha)=\frac{\Delta\left(x v_{m_{\mathrm{E}}}\right)}{\Delta}
$$

and asymmetry

$$
B_{\mathrm{A}}(x ; \alpha)=\frac{\beta\left(x v_{m_{\mathrm{E}}}\right)}{\beta}
$$

functions expressed in terms of the perturber/emitter mass ratio $\alpha=m_{\mathrm{P}} / m_{\mathrm{E}}$ and dimensionless emitter speed $x=v_{\mathrm{E}} / v_{m_{\mathrm{E}}}$, where $v_{m_{\mathrm{E}}}=\sqrt{2 k_{\mathrm{B}} T / m_{\mathrm{E}}}$ is the most probable speed of the emitter.

It should be noted that these functions are dependent on the potentials describing the interactions between emitters and perturbers. The general integral formulae for these functions were given in paper [3]. In the case when the potential is of an inverse-puwer form $V(r)=C_{q} r^{-q}$, the expressions for the functions $B_{\mathrm{W}}(x ; \alpha)$ and $B_{\mathrm{S}}(x ; \alpha)$ were derived in $[13,14]$. One can show that

$$
B_{\mathrm{\Lambda}}(x ; \alpha)=(1+\alpha)^{3 /(2 q-2)} M\left(\frac{3}{2 q-2}, \frac{3}{2},-\alpha x^{2}\right),
$$

where $M(a, b, z)$ is the confluent hypergeometric function. For small $\alpha$, i.e., for light perturbers, the functions $B_{\mathrm{W}}(x ; \alpha), B_{\mathrm{S}}(x ; \alpha)$ and $B_{\mathrm{A}}(x ; \alpha)$ converge to the unity function. In such a case $\gamma_{\mathrm{L}}, \Delta$ and $\beta$ are independent of the emitter velocity.

Figure 1 shows the plots of the functions $B_{\mathrm{A}}(x ; \alpha)$ on $x$, for various $\alpha$. We have performed computations for $\alpha=0.2,1.0,2.0,20.0$ assuming a van der Waals potential: $V(r)=C_{6} r^{-6}$ like for the functions $B_{W}(x ; \alpha), B_{\mathrm{S}}(x ; \alpha)$ in paper [3]. In this case, the function $B_{\mathrm{A}}(x ; \alpha)$ is independent of the force constant $C_{6}$. It should be noted that the functions $B_{\mathrm{W}}(x ; \alpha)$ and $B_{\mathrm{S}}(x ; \alpha)$ are identical in this case and differ very much from the $B_{\mathrm{A}}(x ; \alpha)$ function. This is due to the fact that for the inverse-power potential the width and shift of the line before averaging over perturber and emitter velocities are proportional to $v_{\mathrm{EP}}^{(q-3) /(q-1)}$ in contrast to the asymmetry which is proportional to $v_{\mathrm{EP}}^{-3 /(q-1)}[5]$. 


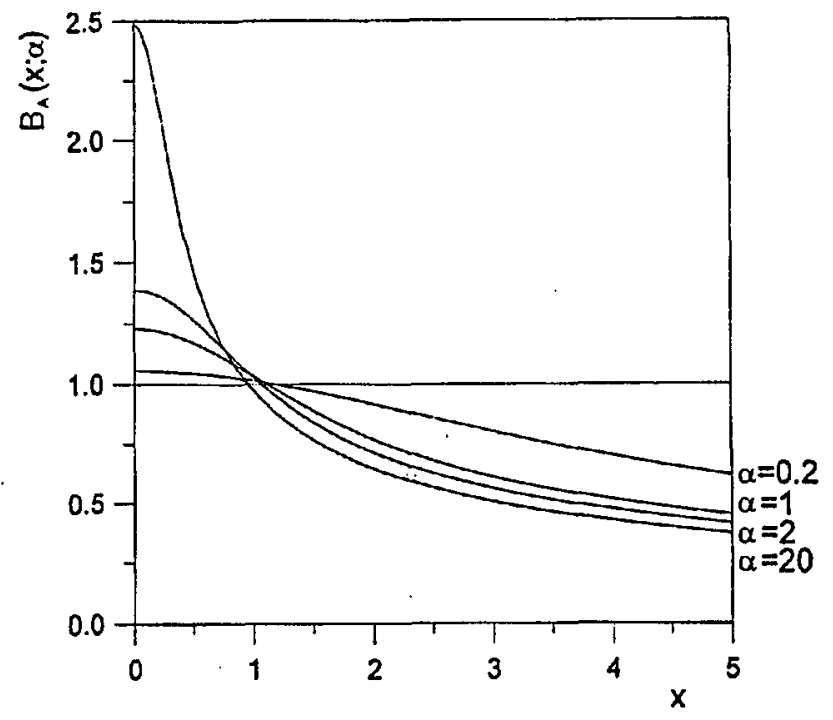

Fig. 1. The plot of $B_{\mathrm{A}}(x ; \alpha)$ for the van der Waals potential and various $\alpha$.

\section{Correlation effects in the combined Doppler and pressure broadening observed by Fabry-Pérot interferometer}

The observed line shape is a convolution of the instrumental function $I_{\mathrm{A}}$ and the real distribution of emitted radiation. If we assume that there is no correlation between collisional and Doppler broadening, then the line profile observed by means of a Fabry-Pérot interferometer is a convolution of the Airy profile and the asymmetric Voigt profile $I_{\mathrm{AvP}}(\omega)$. As was shown in paper [2], such a convolution can be presented in the following analytic form:

$$
\begin{aligned}
I(\omega) & =A\left\{\frac{1}{2}+\sum_{n=1}^{\infty} R^{n} \mathrm{e}^{-n L} \mathrm{e}^{-n^{2} D^{2} / 4}\left[\cos \left(\frac{2 n \pi}{\Omega}\left(\omega-\omega_{0}-\Delta\right)\right)\right.\right. \\
& \left.\left.+\beta \sin \left(\frac{2 n \pi}{\Omega}\left(\omega-\omega_{0}-\Delta\right)\right)\right]\right\},
\end{aligned}
$$

where $A=2 / \Omega, D=\pi \gamma_{\mathrm{D}} / \Omega \sqrt{\ln 2}, L=\pi \gamma_{\mathrm{L}} / \Omega, \gamma_{\mathrm{D}}=\omega_{0} \sqrt{8 k_{\mathrm{B}} T \ln 2 / m_{\mathrm{E}} c^{2}}$ is the Doppler width (FWIM), $R$ is the reflection coefficient of the FPI plates and $\Omega$ is the free spectral range of the Fabry-Pérot interferometer.

To take into account the correlation between the Doppler and collisional broadening, one ought to evaluate a convolution of the speed-dependent asymmetric Voigt profile $I_{\mathrm{SDAvP}}(\omega)$ and the instrumental function. The profile corresponding to such a convolution can be written as [3]

$$
I(\omega)=\int \mathrm{d}^{3} v_{\mathrm{E}} f_{m_{\mathrm{E}}}\left(v_{\mathrm{E}}\right) I_{\mathrm{AL}}\left(\omega-v_{\mathrm{E}} k\right),
$$

where $I_{\mathrm{AL}}(\omega)$ is the convolution of the asymmetric profile $I_{\mathrm{L}}(\omega)$ and the Airy 


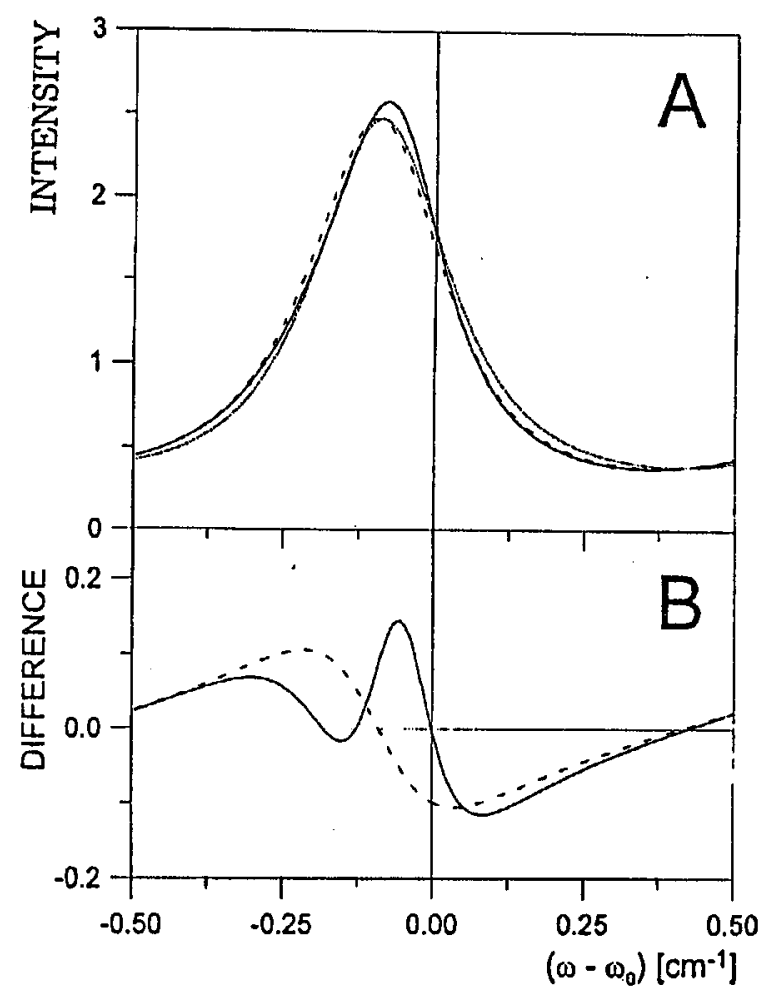

Fig. 2. (A) Comparison of the responses of FPI to SDAVP (full line), AVP (dashed line) and VP (dotted line). (B) The differences of the responses of FPI to SDAVP (full line) and AVP (dashed line) with the responses of FPI to VP. Values of parameters used in this calculations: $\alpha=20, \gamma_{\mathrm{L}}=0.25 \mathrm{~cm}^{-1}, \gamma_{\mathrm{D}}=0.06 \mathrm{~cm}^{-1}, \Delta=-0.09 \mathrm{~cm}^{-1}$, $\beta=0.1, \Omega=1 \mathrm{~cm}^{-1}, R=0.96$.

profile which can be expressed analytically by

$$
I_{\mathrm{AL}}(\omega)=\frac{A}{2} \frac{1-\widetilde{R}^{2}+2 \beta \widetilde{R} \sin \left[\frac{2 \pi}{\Omega}\left(\omega-\omega_{0}-\Delta\right)\right]}{1-2 \widetilde{R} \cos \left[\frac{2 \pi}{\Omega}\left(\omega-\omega_{0}-\Delta\right)\right]+\widetilde{R}^{2}},
$$

where $\widetilde{R}=R \exp (-L)$.

Substituting (14) into (13) and performing calculations similar to those in paper [3], we obtain

$$
\begin{aligned}
& I(\omega)=\frac{2}{\pi^{\frac{3}{2}} \omega_{\mathrm{D}}^{3}} \int_{-\infty}^{+\infty} \mathrm{d} \omega^{\prime} \mathrm{e}^{-\left(\omega^{\prime} / \omega_{\mathrm{D}}\right)^{2}} \omega^{\prime} \\
& \quad \times\left\{\operatorname{att}\left[\frac{1+\widetilde{R}\left(\omega^{\prime} / k\right)}{1-\widetilde{R}\left(\omega^{\prime} / k\right)} ; \frac{\pi}{\Omega}\left(\omega-\omega_{0}-\Delta\left(\omega^{\prime} / k\right)+\omega^{\prime}\right)\right]+\frac{\beta\left(\omega^{\prime} / k\right)}{2}\right. \\
& \left.\quad \times \ln \left[1-2 \widetilde{R}\left(\omega^{\prime} / k\right) \cos \left(\frac{\pi}{\Omega}\left(\omega-\omega_{0}-\Delta\left(\omega^{\prime} / k\right)+\omega^{\prime}\right)\right)+\widetilde{R}^{2}\left(\omega^{\prime} / k\right)\right]\right\},
\end{aligned}
$$


where $\operatorname{att}(a ; x)=\pi$ round $(x / \pi)+\arctan [a \tan (x)]$ and function round $(x)$ rounds a real value $x$ to integer one, $\omega_{\mathrm{D}}=k v_{m_{\mathrm{E}}}, \omega^{\prime}=k v_{\mathrm{E}}, \widetilde{R}\left(\omega^{\prime} / k\right)=R \exp \left[-L\left(\omega^{\prime} / k\right)\right]$, $L\left(\omega^{\prime} / k\right)=\pi \gamma_{\mathrm{L}}\left(\omega^{\prime} / k\right) / \Omega$, and $\gamma_{\mathrm{L}}\left(\omega^{\prime} / k\right)=\gamma_{\mathrm{L}} B_{\mathrm{W}}\left(\omega^{\prime} / \omega_{\mathrm{D}} ; \alpha\right), \Delta\left(\omega^{\prime} / k\right)=$ $\Delta B_{\mathrm{S}}\left(\omega^{\prime} / \omega_{\mathrm{D}} ; \alpha\right), \beta\left(\omega^{\prime} / k\right)=\beta B_{\mathrm{A}}\left(\omega^{\prime} / \omega_{\mathrm{D}} ; \alpha\right)$. In case when $\gamma_{\mathrm{L}}, \Delta$ and $\beta$ are independent of $v_{\mathrm{E}}$, Eq. (15) becomes identical to Eq. (12).

In order to show the differences in the line shape between the response of the Fabry-Pérot interferometer to the speed-dependent asymmetric Voigt profile (15), asymmetric Voigt profile (12) and the ordinary Voigt profile which corresponds to $\beta=0$ in (12) we have performed calculation assuming the interaction potential to be given by the van der Waals form: $V(r)=C_{6} r^{-6}$ and $\alpha=20.0$. Figure 2 shows these three profiles and differences between profiles (15), (12) and the response of FPI to the Voigt profile. It is clearly shown that in the case of the profile (15) deviations from the response of FPI to the Voigt profile are caused by the correlation effects in the core of the line and by the collision-time asymmetry in line wings. As is seen in Fig. 3 dispersion parts of the profiles (15) and (12) differ from each other, but the difference between them is not great and decreases with decreasing $\alpha$.

As is shown in Fig. 2 the asymmetry of the spectral line can be caused by the finite time of collisions (the dispersion part of the profile (1)) and also by correlations between the pressure and Doppler broadening, that means by dependence of the pressure line shift on the emitter velocity. It should be emphasized, however, that at intermediate pressures of the perturbing gas the effect due to the finite duration of collision occurs simultaneously with that due to the correlations

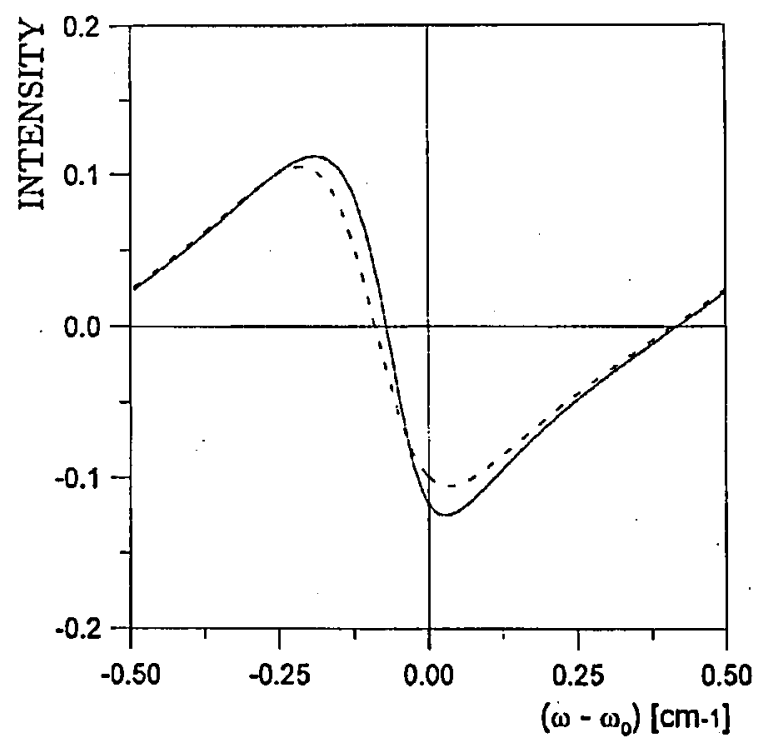

Fig. 3. Comparison of the dipersion parts of the responses of FPI to SDAVP (full line) and AVP (dashed line). All parameters are the same as in Fig. 2. 
between the Doppler and collisional broadening provided the perturber mass is heavy enough, i.e. when the perturber mass is greater or comparable with the mass of the emitter (cf. also [10-12]). In such a case it is necessary to take into account the simultaneous occurrence of these two effects in order to perform a correct interpretation of experimentally observed line profiles. Equation (15) derived in the present paper may thus be useful in the analysis of experimental line shapes obtained by means of the scanned Fabry-Pćrot interferometer when both the collision-time and the Doppler-collision correlation asymmetry effects should be simultaneously included.

\section{Acknowledgment}

When this work was done, one of authors (R.C.) was the scholarship-holder of the Foundation for Polish Science. This work was supported by a grant No. 673/P03/96/10 (2 P03B 005 10) from the Committee for Scientific Research.

\section{References}

[1] E.A. Ballik, Appl. Opt. 5, 170 (1966).

[2] A. Bielski, R. Bobkowski, J. Szudy, Appl. Opt. 27, 3864 (1988).

[3] R. Ciuryło, A. Bielski, S. Brym, J. Jurkowski, J. Quant. Spectrosc. Radiat. Transf. 53, 493 (1995).

[4] J. Szudy, W.E. Baylis, J. Quant. Spectrosc. Radiat. Transf. 15, 641 (1975).

[5] J. Szudy, W.E. Baylis, J. Quant. Spectrosc. Radiat. Transf. 17, 681 (1977).

[6] A. Royer, Acta Phys. Pol. A 54, 805 (1978).

[7] P.S. Julienne, F.II. Mies, Phys. Rev. A 34, 3792 (1986).

[8] R.E. Walkup, A. Spielfiedel, D.E. Pritchard, Phys. Rev. Lett. 45, 986 (1980).

[9] R.E. Walkup, B. Stewart, D.E. Pritchard, Phys. Rev. A 29, 169 (1984).

[10] M. Harris, E.L. Lewis, D. McIIugh, I. Shannon, J. Phys. B 17, L661 (1984).

[11] I. Shannon, M. IIarris, D. McIIugh, E.L. Lewis, J. Phys. B 10, 1409 (1986).

[12] M. Harris, E.L. Lewis, D. McIIugh, I. Slannon, J. Phys. B 19, 3207 (1986).

[13] P.R. Berman, J. Quant. Spectrosc. Radiat. Transf. 12, 1331 (1972).

[14] J. Ward, J. Cooper, E.W. Smith, J. Quant. Spectrosc. Radiat. Transf. 14, 555 (1974). 